\title{
Neurological complications in benign parapharyngeal space tumors - Systematic review and meta-analysis
}

\author{
Muhammad Faisal ${ }^{1}$, Rudolf Seemann ${ }^{2}$, Gregor Fischer ${ }^{3}$, Claudia Lill ${ }^{2}$, Sasan Hamzavi², \\ Arno Wutzl ${ }^{2}$, Arif Jamshed ${ }^{1}$, Raza Hussain ${ }^{1}$, and Boban Erovic ${ }^{2}$ \\ ${ }^{1}$ Shaukat Khanum Memorial Cancer Hospital and Research Centre \\ ${ }^{2}$ Evangelisches Krankenhaus \\ ${ }^{3}$ Danube University Krems
}

May 8, 2020

\begin{abstract}
Background: Parapharyngeal space tumors with complex anatomy and diverse histology and have remained a challenging phenomenon for treating physicians. Objective: Assess the factors for selection of surgical approach and association of neurological complications with tissue of origin in parapharyngeal space tumors. Type of review: Systematic review and meta-analysis of retrospective studies in accordance with PRISMA guidelines Methods: We have conducted a comprehensive web search on Pubmed, Web of Science, EMBASE, Cochrane Library, Biomedical Literature Database (CBM) and Clinicaltrials.gov. Two researches reviewed all identified articles independently with a third reviewer for adjudication. Patient demographics and other clinicopathological characteristics were explored. Main outcome measures: Primary outcomes assessed were post-operative complications i.e. neurological and salivary, surgical approaches used and the factors used for the selection of surgical approaches. Results: The systematic review has identified 631 patients of benign parapharyngeal space tumors with neurogenic and salivary tissue histology in 13 studies with a mean age of $42.9 \pm 7.76$ years and a median follow-up of $40.98 \pm 19.1$ months. Salivary gland $(50.8 \%)$ and neurogenic $(49.1 \%)$ tumors were the most common histological entities. Tumor size, location, histology, deep parotid lobe involvement and proximity to great vessels or skull base were the deciding factors in selecting the surgical approach. Factors considered to select surgical approach do not seem to have a correlation with the outcome in terms of neurological sequalae $(\mathrm{p}=0.106)$. Neurological deficit was observed in $48 \%$ of patients with neurogenic histology (148/310) while only $13 \%$ patients with salivary tumor developed neurological deficit. The pooled RR was 2.41 (95\% CI $1.80-3.23$, p=0.001). Conclusion: Neurologic complications are significantly associated with neurogenic benign tumors rather than surgical approach.
\end{abstract}

\section{Key points:}

- There are many published series and reports regarding surgical management of parapharyngeal space tumors mentioning neurological complications.

- The reported incidence of neurological deficit is high in both benign and malignant tumors.

- We have performed a comprehensive review of literature over the last 30 years (1989-2019).

- This systematic review and meta-analysis has revealed that tissue of tumor origin (salivary vs neurogenic) is the key factor contributing towards neurological complications.

- This preemptive assessment of anticipated range of neurologic complications must be considered to educate the patients on the post-operative sequelae and simultaneous preparation to facilitate rehabilitation.

\section{Introduction:}

Parapharyngeal space (PPS) is an imaginary inverted pyramidal space with an extension from skull base to the hyoid bone divided by Riolan's bundle into pre- and post-styloid compartments [1-3]. Of all head 
and neck cancers, PPS tumors account for $0.5 \%$ and mostly are benign (80\%) with diverse histology. Pleomorphic adenoma is the most common benign tumor of salivary gland origin occupying mostly prestyloid compartment and Schwannomas as the most common neurogenic entity residing in the post-styloid space [4]. The correct histopathological diagnosis of these lesions may not be achieved due to complex anatomical location relying more on image findings (Computerized tomography, Magnetic resonance imaging or Angiography) for preliminary diagnosis. This may also help in identifying the anatomical extent of the growth [1]. Surgery has stayed as the primary treatment modality if not contraindicated by gross intracranial tumor extension or co-morbidities pushing towards other non-surgical treatment options [5]. A lot of literature including case series and reports has been published regarding parapharyngeal space tumors with diverse benign and malignant histology including all the deep lobe parotid, infra-temporal fossa tumors as well as carotid body paragangliomas. [6]. Previous published data have reported neurological complications to be a common observation among PPS tumors [5-7]. We have aimed our study more on the tissue of tumor origin in benign parapharyngeal space tumors and its relation to neurological deficit. A thorough search was conducted by reviewing all the available relevant published data to focus on the rationale behind high incidence of associated neurological complications.

\section{Materials and Methods:}

\subsection{Literature search for data collection}

We have performed extensive search through PubMed, Cochrane Library, Web of Science, EMBASE, Biomedical Literature Database (CBM) and Clinicaltrials.gov with reference to PPS tumors. Articles meeting the search criteria with keywords such as 'parapharyngeal space' or 'transmandibular' or 'benign parapharyngeal space tumor' or 'trans-cervical' or 'lip split mandibulotomy' or 'mandibular swing' were included in the first instance and concluded on 25.05.2019. The analysis included systematic reviews, retrospective studies, literature reviews pertaining to open surgical management of primary parapharyngeal space tumors published from 1989 to 2019. No focused publication was found on the management of benign parapharyngeal space tumors due to rarity of the disease. The studies with management of parapharyngeal space tumors including series with patients not less than 10 were also incorporated. Data was collected including patient's age, gender, year of publication, histopathological classification, surgical approaches and complications. The criteria for exclusion was case reports / series addressing malignant pathology only, treatment modalities other than surgery, single case reports or case series having less than 5 patients, non-primary parapharyngeal space tumors, tumors with nasopharyngeal, intracranial, infratemporal fossa extensions, studies comparing approaches such as robotic / lateral skull, studies that provide data using single modality with no comparisons, letter to the editors, meeting abstract and editorials.

Robotic and endoscopic approaches have not been included in the review as majority of the published literature have addressed PPS tumors with open surgical access. Further, the endoscopic approaches may still need to be validated for reproducibility and effectiveness to be labelled as the standard of care. We had to incorporate different benign histologies as the numbers are very few and surgical management of these tumors have been based more on the location of the tumor rather than tissue of origin.

\subsection{Data extraction}

Data extraction and quality assessment of all included studies were independently performed by 2 authors (MF and BE). Controversies were solved by discussion or consultation with another author (RS). Basic information such as patient demographics, histopathology, presenting complaints, surgical approaches and post-operative complications were recorded. All included papers were clinical studies focusing on surgical management of PPS tumors using different approaches and the post-operative complication or sequelae.

\subsection{Data analysis and Statistical methods}

SPSS $($ ) version 25 (IBM, Armonk, NY, USA) was used for statistical analysis. Independent T test was used to compare the means of 2 normally distributed groups (salivary vs neurogenic tumors). A Pearson correlation coefficient was calculated to determine the Correlation of tumor' $\mathrm{s}$ tissue of origin and determinant 
factors for selecting surgical approach to the post-operative complications. Binary logistic regression analysis was performed to measure the odds of having neurological complications in salivary and neurogenic PPS tumors. A p-value $<0.05$ was significant. GraphPad Prism 8 (GraphPad Software, San Diego, California, USA) was used to draw the bar charts. The free available software RevMan 5.3 (Cochrane Collaborative, Oxford, England) was used for the meta-analysis and creation of the forest plot. The risk ratios (RRs) of neurological complications and their $95 \%$ confidence intervals (CIs) were calculated for each study. Statistical heterogeneity was assessed using the Cochran $Q$ statistic ( $P$ value for heterogeneity) and the $I$ 2 statistic (total percentage of variation resulting from heterogeneity). In case of significant heterogeneity $(I$ 2 [?] 50) the random-effect model was used, while the fixed-effect model was used in absence of significant heterogeneity. Herein, we solely applied the fixed-effect model to obtain RR, HR, 95\% CI, and $P$-value.

\section{Results:}

3.1 Search findings A total of 2674 articles were identified with the database search using keywords as mentioned in methodology section. 1852 articles remained after duplication removal. Next, the articles were screened by title and abstract reading to select the relevant studies and 745 remained at this step. The short case series reporting less than 5 patients were further excluded. After full-text revision, 679 articles were excluded for reasons described in Plasma flow chart. Hence, 13 studies all published in English, with 631 participants were included for further evaluation [6-18]. We followed the PRISMA guidelines, and the study selection procedure was illustrated by the PRISMA flow diagram (Figure 1). Three studies were conducted in USA, two in Spain, two in China and one each in Egypt, India, Israel, Brazil, Italy and Japan [6-18]. Six hundred and thirty one (631) patients were recruited from studies published between 1996 - 2017.

\subsection{Study cohort}

There were 631 patients with benign histology (either salivary or neurogenic in origin). The mean age (in years) at presentation was $42.9+-7.76$ with a median follow up of $40.98+-19.1$ months. Baseline characteristics and demographics along with presenting complaints, histological variation and complications of cumulative data have been further expressed (Table 1). The patients were categorized into two groups based on the tissue of origin such as neurogenic $(n=310)$ and salivary $(321)$, later compared on the basis of subsequent complications. The primary surgical approached used were (i) Trans-cervical (60\%), (ii) Cervicoparotid (30\%) including dissection and exposure of the facial nerve trunk and associated branches and (iii) Trans-mandibular (10\%) comprising of mandible split for access and tumor resection (Figure 2). Transcervical approach was favored in 9 out of 13 studies as compared to 4 studies where cervico-parotid access was advocated. None of the studies has given clear preference to the use of trans-mandibular approach. The most common neurogenic tumor found was schwannoma (54\%) followed by paraganglioma (43\%) (Figure 2). The distribution of complications based on salivary or neurogenic histology has been represented for all the studies included in the review.

The 148 patients with neurogenic complication among 310 neurogenic tumors (Mean 11.38, SD 11.81) were compared against 41 patients with neurological complication among 321 salivary gland tumors and demonstrated significantly increased rate of nerve related complication $(t=2.42, p<0.05)$ (Figure 3 ). Among the neurologic complications, most encountered were vocal cords palsy $(73 \%)$, Horner syndrome (9\%), hypoglossal nerve injury (6\%) and first bite syndrome (4\%) (Table 2).

Factors considered for selecting surgical approaches in 13 studies on PPS tumors were categorized into size, location, histology, proximity to greater vessels or base of skull, histology and deep parotid lobe involvement (Table 2). Each factor was given a score and the combined scores were compared to the percentage of complications to assess if any of these factors considered before surgery would have an effect in the outcome in terms of complication rate. The correlation coefficient suggested a small degree of positive correlation that is not statistically significant $(\mathrm{p}=0.930)$. The tissue of origin (neurogenic vs. salivary) seems to have a positive correlation with more complications related to neurogenic tumors (Pearson coefficient 0.662, $\mathrm{p}=0.019$ ) (Figure 4). The binary logistic regression showed the significantly increased likelihood of developing post-operative neurological complications in tumors with neurogenic histology $(\mathrm{p}=0.0001)$ (Table 3$)$. 
Next we performed a meta-analysis assessing neurological complications among the groups having salivary or neurogenic tissue of origin. Due to heterogeneity $<50 \%$, fixed term model was used. Data of 631 patients was available for analysis of whom 321 were of salivary tissue origin and 310 were neurogenic in histology. Neurological deficit was observed in $48 \%$ of patients with neurogenic histology (148/310) while only $13 \%$ patients with salivary tumor developed neurological deficit. The pooled RR was 2.41 (95\% CI $1.80-3.23$, $\mathrm{p}=0.001)$ (Figure 5).

\subsection{Quality of studies}

The risk of bias of included works has been assessed in eight categories using the ACROBAT-NRSI tool recommended by the Cochrane group and an over-all score was calculated indicating the quality of each analyzed study (Table 4). Altogether, eleven studies with a moderate risk, and two studies were categorized with a serious risk of bias. In particular, $3(23 \%)$ and $5(38 \%)$ studies were rated with a high risk of bias in the categories" match with prognostic variables" and "Co-intervention between groups".

\section{Discussion:}

Benign tumors are the most common entity (80-90\%) found in parapharyngeal space (PPS) with parotid gland as the most common site of origin [19, 20-21]. The parapharyngeal space is divided into 2 compartments by an osteomuscular aponeurotic sheath originating at the styloid process. The pre-styloid space contains components such as deep lobe of the parotid gland, fat and lymphatic nodes, inferior, lingual and temporal auricular alveolar nerves. The retro-styloid space encloses neurovascular structures such as carotid artery, jugular vein, cervical sympathetic chain, cranial nerves IX, X, XI and XII suggesting more diverse histology from this compartment [22]. Parapharyngeal space tumors must be clearly identified as erroneous anatomical boundaries may have resulted in inclusion of adjacent tumors in some of the published literature such as inclusion of deep lobe tumors should only be considered if the location is retro-mandibular. Similarly, lesions at foramen ovale must be a part of infratemporal fossa tumors while carotid body paragangliomas below the posterior body of digastric must not be considered a part of PPS tumors [23]. Due to complex anatomical location and diverse histology, precise approaches to the PPS tumors have remained a controversial debate $[24,25]$. Complete excision with minimal morbidity should be the aim of the operating surgeon particularly when dealing with benign PPS tumors. Size, location (pre / post styloid), proximity to the skull base and vascular bundle, extension to the deep lobe of the parotid and imaging based position (superior/middle/inferior) of the lesions are some of the key factors considered pre-operatively to select a surgical approach $[7,13,15]$.

The inclusion of mandibulotomy have raised many concerns such as facial scar, oro-cervical fistula, prolonged operating time, malocclusion, trismus, delay in resuming regular diet and risk for exposure of fixation plate and temporomandibular joint (TMJ) dysfunction [26-28]. The most used approach to access PPS tumors published in literature has been trans-cervical either alone or in combinations. In the cumulative series of 631 patients, this approach has been utilized for $60 \%$ of cases addressing both salivary and extra parotid neurogenic tumors. The facial nerve may not need to be exposed to further extend the dissection and exposure. Due to excessive push and pull, neuropraxia may occur in the post-operative setting. The facial nerve identification may increase the access to large sized and superiorly placed PPS tumors. The rationale behind the use of trans-cervical approach in our cumulative search was small (preferably less than $8 \mathrm{~cm}$ ), benign, pre-styloid extra parotid tumors in the lower parapharyngeal space. Further exposure can be achieved by division of posterior belly of digastric muscle and removal of submandibular gland on occasions.

The cervico-parotid approach uses formal identification of facial nerve trunk and indicated for tumors in proximity to the deep lobe of the parotid gland having the risk of adherence to the facial nerve. Transcervical approach only may jeopardize the preservation of facial nerve. In our cumulative results, it is the second most used approach well in accordance with previous published data. The most commonly used indications in the data for cervico-parotid access were large, pre or retro-styloid, deep lobe parotid or minor salivary gland as well as neurogenic tumors with or without facial nerve involvement locating middle to lower PPS but not involving the base of skull. Access may be further enhanced by prognathic mandibular 
dislocation dividing the stylomandibular ligament and styloid muscle.

The transmandibular approach with its modifications (Midline / paramedian or lateral splits) is usually performed for massive, large vascular or recurrent benign tumors placed more superiorly in the PPS. In the cumulative series, transmandibular approach was only used in $6 \%$ of the patients and more so because of the large size (mostly $>8 \mathrm{~cm}$ ), vascular nature of the tumor, superior location in the PPS and proximity to skull base. The use of transmandibular approach has been further limited as indicated by the recent studies showing decline in the use of transmandibular approach (9\% to $6 \%$ ) when compared with previous reports [29-31]. Prolonged hospital stays, delay in resuming normal diet, tracheostomy covering, trismus, associated TMJ and Occlusal disturbances are some of the drawbacks of this technique [32, 33].

The cumulative studies in our cohort have depicted significantly high number of neurogenic complications in both salivary and neurogenic tumors combined. Detailed analysis has shown these complications to be more common in tumors with neurogenic tissue of origin. Literature has described five major histological subtypes of neurogenic tumors with more than $90 \%$ having benign histology. Riffat et al. have reported paraganglioma to be the most common subtype while John et al. and Danke et al. have found Schwannomas to be the commonly found histological entity [34, 35]. Schwannomas arise usually from cranial nerves IX to XII or cervical sympathetic chain with decreased risk of nerve injury when small in size. On the other hand, paragangliomas are derived from vagal nerve with a potential for intracranial extension or malignant transformation. The list of complications in our combined series included vocal cord paralysis (73\%), Horner's syndrome $(9.3 \%)$, hypoglossal nerve injury $(6.7 \%)$ and first bite syndrome $(4 \%)$. The tumors with salivary histology had a complication rate $12.8 \%$ as compared to neurogenic tumors $(47.7 \%)$ which is significantly lower than neurogenic tumors $(\mathrm{t}$ value $=2.42, \mathrm{p}=0.023$ ). This preemptive assessment of anticipated range of neurologic complications must be considered to educate the patients on the post-operative sequelae and simultaneously preparation to facilitate rehabilitation.

Our systematic analysis may have its share of limiting factors. Most of the studies included are retrospective series with inherent biases for selection of surgical approaches, clinical expertise, intraoperative and postoperative care with a variable support for post-operative rehabilitation. Majority of studies have omitted data on some critical factors when applying these surgical approaches such as length of hospital stay, cost effectiveness, return to normal diet or oral feeding in trans-mandibular approach, infection, time to healing and length of the surgery.

\section{Conclusion:}

The majority of parapharyngeal space tumors are benign $(80 \%)$ having either neurogenic or salivary origin. Cranial nerve deficits are the result of complications arising from the neurogenic tissue during surgery irrespective of which surgical approach is used. The physicians need to establish rehabilitation protocols to avoid the severity of functional compromise post-surgery

\section{References:}

1. Metgudmath RB, Metgudmath AR, Malur PR, Das AT. Surgical management of parapharyngeal space tumors: our experience. Indian J Otolaryngol Head Neck Surg. 2013;65:64-68.

2. Bradley PJ, Bradley PT, Olsen KD. Update on the management of parapharyngeal tumours. Curr Opin Otolaryngol Head Neck Surg. 2011;19:92-98.

3. Olsen KD. Tumors and surgery of the parapharyngeal space. Laryngoscope. 1994;104:1-28.

4. Singh M, Gupta S, Singla A. Our experiences with parapharyngeal space tumors and systematic review of the literature. Indian J Otolaryngol Head Neck Surg. 2009;61:112-119.

5. Luna-Ortiz K, Navarrete-Aleman JE, Granados-Garcia M, et al. Primary parapharyngeal space tumors in a Mexican cancer center. Otolaryngol Head Neck Surg. 2005;132:587-591.

6. Grilli G, Suaarez V, Munoz MG, Costales M, Llorente JL. Parapharyngeal space primary tumours. Acta Otorrinolaringol Esp. 2017;68:138-144.

7. Cohen SM, Burkey BB, Netterville JL. Surgical management of parapharyngeal space masses. Head Neck. 2005;27:669-675. 
8. Attia A, El-Shafiey M, El-Shazly S, Shouman T, Zaky I. Management of parapharyngeal space tumors at the National Cancer Institute, Egypt. J Egypt Natl Canc Inst. 2004;16:34-42.

9. Miller FR, Wanamaker JR, Lavertu P, Wood BG. Magnetic resonance imaging and the management of parapharyngeal space tumors. Head Neck. 1996;18:67-77.

10. Hazarika P, Dipak RN, Parul P, Kailesh P. Surgical access to parapharyngeal space tumours-the Manipal experience. Med J Malaysia. 2004;59:323-329.

11. Zhi K, Ren W, Zhou H, Wen Y, Zhang Y. Management of parapharyngealspace tumors. J Oral Maxillofac Surg. 2009;67:1239-1244.

12. Malone JP, Agrawal A, Schuller DE. Safety and efficacy of transcervical resection of parapharyngeal space neoplasms. Ann Otol Rhinol Laryngol. 2001;110:1093-1098.

13. Khafif A, Segev Y, Kaplan DM, Gil Z, Fliss DM. Surgical management of parapharyngeal space tumors: a 10-year review. Otolaryngol Head Neck Surg. 2005;132:401-406.

14. Tincani AJ, Martins AS, Altemani A, et al. Parapharyngeal space tumors: considerations in 26 cases. Sao Paulo Med J. 1999;117:34-37.

15. Cassoni A, Terenzi V, Della Monaca M, et al. Parapharyngeal space benigntumours: our experience. J Craniomaxillofac Surg. 2014;42:101-105.

16. Iglesias-Moreno MC, Lopez-Salcedo MA, Gomez-Serrano M, et al. Parapharyngeal space benign tumours: fifty-one cases managed in a single tertiary care center. Acta Otolaryngol (Stockh). 2015;20:1-6.

17. Ijichi Kei, Murakami Shingo. Surgical treatment of parapharyngeal space tumors: a report of 29 cases. Oncol Lett. 2017;14:3249-3254.

18. Shi X, Tao L, Li X, et al. Surgical management of primary parapharyngeal space tumors: a 10-year review. Acta Otolaryngol. 2017;137:656-661.

19. Hughes KV, Olsen KD, McCaffrey TV: Parapharyngeal space neoplasms. Head Neck. 1995;17:124-130.

20. Carrau RL, Meyers EN, Johnson JT: Management of tumors arising in the parapharyngeal space. Laryngoscope. 1990;100:583-589.

21. Luksic I, Virag M, Manoijlovic S, Macan D: Salivary gland tumours: 25 years of experience from a single institution in Croatia. J Craniomaxillofac Surg. 2012;40:75-81.

22. Batsakis JG, Sneige N. Pathology consultation: parapharyngealand retropharyngeal space diseases. Ann Otol Rhinol Laryngol. 1989;98:320-321.

23. Curtin HD. Separation of the masticator space from the parapharyngeal space. Radiology. 1987;163:195-204.

24. Scheithauer MO, Bohn JC, Riechelmann H. Median sagittal mandibulotomy in head-neck tumors. Laryngo Rhino Otol. 2000;79:490-497.

25. Gooris PJ, Worthington P, Evans JR. Mandibulotomy: a surgical approach to oral and pharyngeal lesions. Int J Oral Maxillofacial Surgery. 1989;18:359-364.

26. Li W, Li R, Safdar J, Huang S, Xu Z, Tan X. Modified visor approach applied to total or subtotal glossectomy and reconstruction: avoidance of lip splitting and mandibulotomy and cutting off mental nerve. Tumor Biol. 2014;35:7847-7852.

27. Eisen MD, Weinstein GS, Chalian A, et al. Morbidity after midline mandibulotomy and radiation therapy. Am J Otolaryngol. 2000;21:312-317.

28. El-Zohairy MA. Straight midline mandibulotomy: technique and results of treatment. J Egyptian Natl Cancer Inst. 2007;19:292-298.

29. Riffat F, Dwivedi RC, Palme C, Fish B, Jani P. A systematic review of 1143 parapharyngeal space tumors reported over 20 years. Oral Oncol. 2014;50:421-430.

30. Kuet ML, Kasbekar AV, Masterson L, Jani P. Management of tumors arising from the parapharyngeal space: a systematic review of 1293 cases reported over 25 years. Laryngoscope. 2014;125:1372-1381.

31. Sheahan P. Surgical approaches to the parapharynx and nasopharynx, transcervical approach for removal of benign parapharyngeal space tumors. Op Tech Otolaryngol. 2014;25:227-233.

32. Abdel-Haleem A, El Sayed A, Hakeem HA. Transmandibular approach in parapharyngeal tumors; when to do it? Egypt J Ear Nose Throat Allied Sci. 2011;12:25-31.

33. Chu F, Tagliabue M, Giugliano G, Preda L, Ansarin M. From transmandibular to transoral robotic 
approach for parapharyngeal space tumors. Am J Otolaryngol. 2017;38:375-379.

34. John DG, Carlin WV, Brown MJ. Tumours of the parapharyngeal space. J R CollmSurg Edinb 1988;33(2):56-60.

35. Dankle SK. Neoplasms of the parapharyngeal space. Ear Nose Throat J 1987;66(12):491-501.

Figure Legends:

Figure 1: Flow diagram; the flow diagram was adapted according to the PRISMA recommendations

Figure 2: Distribution of PPS tumors based on surgical approach and tumor histology

Figure 3: Comparison of complication rates based on tissue of origin in PPS tumors

Figure 4: Correlation of surgical approaches and neurogenic histology to complications in PPS tumors

Figure 5: Meta-analysis of neurological complications in parapharyngeal space tumors

Hosted file

Table-1.docx available at https://authorea.com/users/319547/articles/449284-neurologicalcomplications-in-benign-parapharyngeal-space-tumors-systematic-review-and-meta-analysis

Hosted file

Table-2.docx available at https://authorea.com/users/319547/articles/449284-neurologicalcomplications-in-benign-parapharyngeal-space-tumors-systematic-review-and-meta-analysis

Hosted file

Table-3.docx available at https://authorea.com/users/319547/articles/449284-neurologicalcomplications-in-benign-parapharyngeal-space-tumors-systematic-review-and-meta-analysis

Hosted file

Table 4.docx available at https://authorea.com/users/319547/articles/449284-neurologicalcomplications-in-benign-parapharyngeal-space-tumors-systematic-review-and-meta-analysis

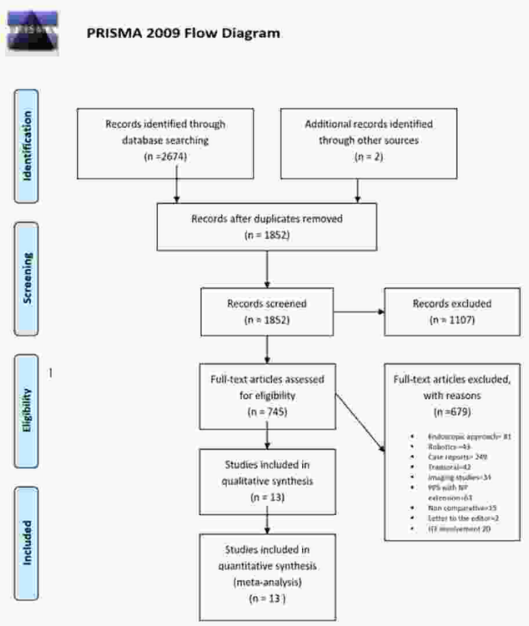




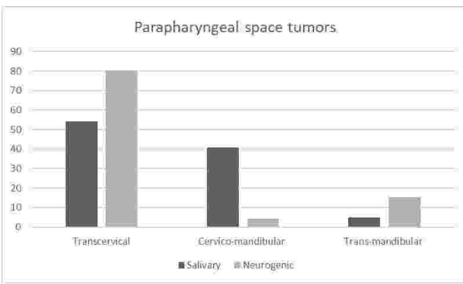

A Distribution of surgical approaches based on tissue of tumor origin

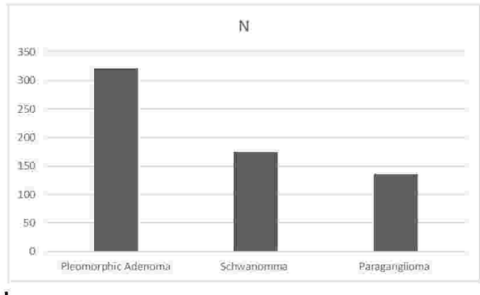

b Distribution of PPS tumor histology based on salivary and neurogenic tissue
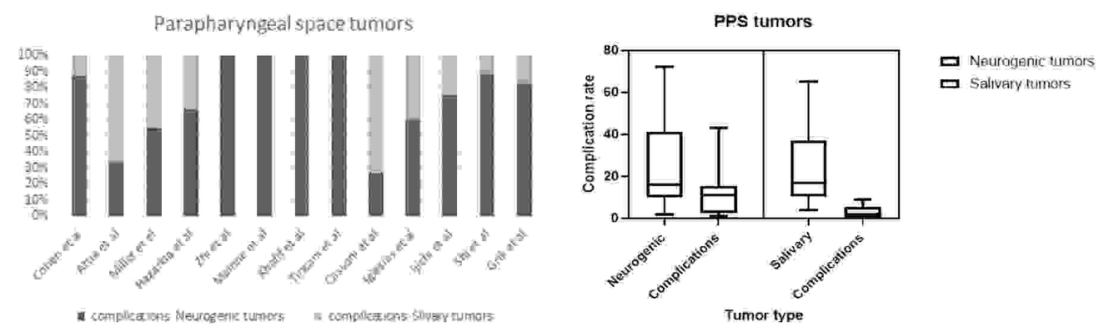

A Distribution of salivary and neurogenic histology among selected studies

b comparison of complication rates in neurogenic and salivary gland tumors bases on tissue of origin 


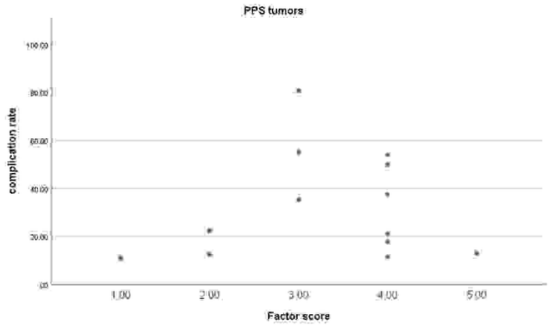

a correlation between determinants for selection

of surgical approach and complication rate

(Correlation coefficient .027, $\mathrm{p}=0.930$ )

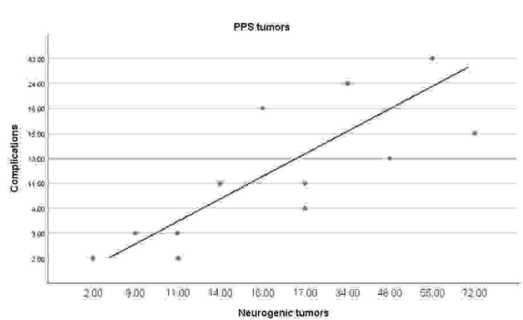

b Positive correlation of Neurogenic tumor histology with associated neurological complications in PPS tumors (Pearson correlation $=0.686, p=0.019$ )

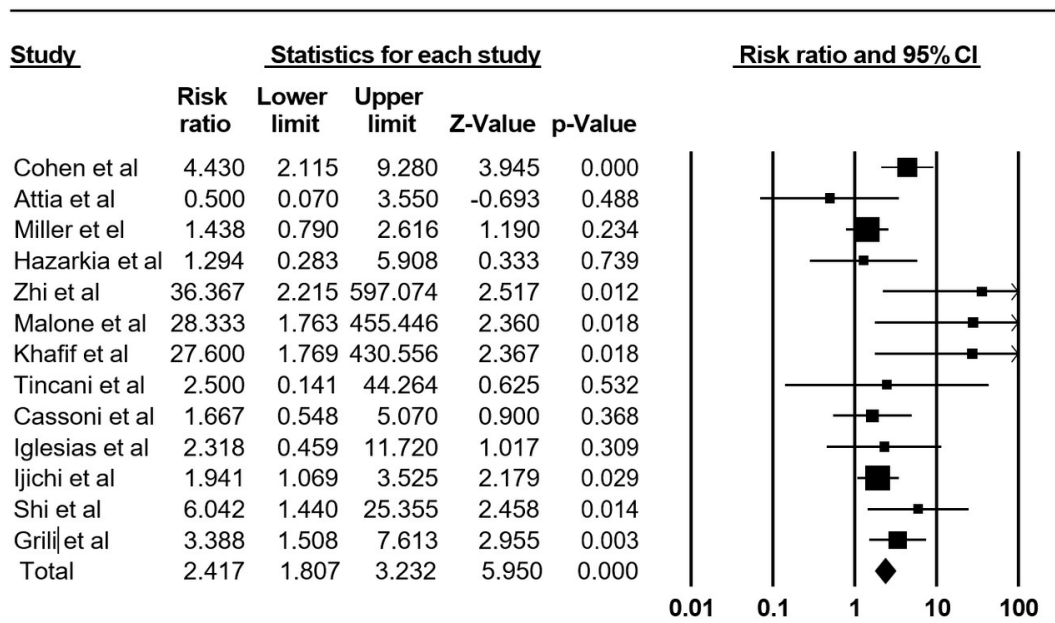

Favors Neurogenic Favors Salivary

Heterogeneity: $\mathrm{Tau}^{2}=0.871 ; \mathrm{Chi}^{2}=23.8 ; \mathrm{df}=12(0.021) ; \mathrm{I}^{2}=49.6 \%$

Test for overall effect: $z=7.244(p=0.001)$ 